\title{
Observational Properties of Thermonuclear Supernovae
}

\author{
Saurabh W. Jha ${ }^{1,2}$, Kate Maguire ${ }^{3,4}$, Mark Sullivan ${ }^{5}$
}

August 7, 2019

authors' version of Nature Astronomy invited review article

final version available at http://dx.doi.org/10.1038/s41550-019-0858-0

\begin{abstract}
${ }^{1}$ Department of Physics and Astronomy, Rutgers, the State University of New Jersey, Piscataway NJ, USA ${ }^{2}$ Center for Computational Astrophysics, Flatiron Institute, New York, NY, USA ${ }^{3}$ Astrophysics Research Centre, School of Mathematics and Physics, Queen's University Belfast, UK ${ }^{4}$ School of Physics, Trinity College Dublin, Ireland ${ }^{5}$ School of Physics and Astronomy, University of Southampton, Southampton, SO17 1BJ, UK
\end{abstract}

The explosive death of a star as a supernova is one of the most dramatic events in the Universe. Supernovae have an outsized impact on many areas of astrophysics: they are major contributors to the chemical enrichment of the cosmos and significantly influence the formation of subsequent generations of stars and the evolution of galaxies. Here we review the observational properties of thermonuclear supernovae, exploding white dwarf stars resulting from the stellar evolution of low-mass stars in close binary systems. The best known objects in this class are type Ia supernovae (SN Ia), astrophysically important in their application as standardisable candles to measure cosmological distances and the primary source of iron group elements in the Universe. Surprisingly, given their prominent role, $\mathrm{SN}$ Ia progenitor systems and explosion mechanisms are not fully understood; the observations we describe here provide constraints on models, not always in consistent ways. Recent advances in supernova discovery and follow-up have shown that the class of thermonuclear supernovae includes more than just SN Ia, and we characterise that diversity in this review.

The modern classification scheme for supernovae traces back to Minkowski ${ }^{1}$ who in 1941 split "Type I" from "Type II" supernovae based on optical spectra. Further subdivision of these basic classes has continued on an empirical basis ${ }^{2,3}$, and in our review we describe the observational properties of what are now called SN Ia, along with other similar objects. The observational classification effort arises from a desire for physical understanding of these objects, explaining our use of the term thermonuclear supernovae in the title. That categorisation is based on the explosion mechanism: objects where the energy released in the explosion is primarily the result of thermonuclear fusion. Given our current state of knowledge, we could equally well call this a review of the observational properties of white dwarf supernovae, a categorisation based on the kind of object that explodes. This is contrasted with core-collapse or massive star supernovae, respectively, in the explosion mechanism or exploding object categorisations. Unlike those objects, where clear observational evidence exists for massive star progenitors and corecollapse (from both neutrino emission and remnant pulsars), the direct evidence for thermonuclear supernova explosions of white dwarfs is limited $d^{4,5}$ and not necessarily simply interpretable ${ }^{6,7}$. Nevertheless, the indirect evidence is strong, though many open questions about the progenitor systems and explosion mechanisms remain.

SN Ia are important both to the evolution of the Universe and to our understanding of it. As standardisable candles whose distance can be observationally inferred ${ }^{8}$, SN Ia have a starring role in the discovery of the accelerating expansion of the Universe ${ }^{9,10}$ and in measurement of its current expansion rate ${ }^{11}$. SN Ia are also major contributors to the chemical enrichment of the Universe, producing most of its iron ${ }^{12}$ and elements nearby in the periodic table. Because of the stellar evolutionary timescales involved, the enrichment of these elements occurs differently from other elements whose main origin is in massive star supernovae.

Here we review the observational properties of thermonuclear supernovae, including both normal SN Ia and related objects. We describe the photometric and spectroscopic properties of SN Ia in section 1, and their environments and rates in section 2. Evidence has been growing that not all thermonuclear explosions of white dwarfs result in "normal" SN Ia; we discuss related supernovae in section 3. In this review article we provide a broad overview supplemented by further discussion of the newest developments. Our reference list is limited and thus necessarily incomplete. We have chosen to highlight illustrative, recent works with a strong bias towards observations rather than theory or models. These deficiencies are rectified in recent reviews that cover many of these topics in more detail ${ }^{13-15}$. 


\section{Type la Supernovae}

Energetics and light curve properties: The runaway thermonuclear explosion of a carbon-oxygen white dwarf to irongroup elements releases on the order of $10^{51}$ erg as kinetic energy that unbinds the star. The expanding ejecta travel at $\sim 10,000 \mathrm{~km} \mathrm{~s}^{-1}$ and cool rapidly. The luminosity of SN Ia is subsequently powered by the decay of radioactive elements that were synthesised in the explosion ${ }^{16,17}$. The primary power source is the isotope nickel-56, which decays to cobalt-56 with a half-life of 6.1 days, and which in turn decays with a half-life of 77.3 days to stable iron-56. The peak SN Ia bolometric luminosity is typically of the order of $10^{43} \mathrm{erg} \mathrm{s}^{-1}$, with $0.3-0.8 \mathrm{M}_{\odot}$ of iron-56 ultimately produced in each event. The majority $(\sim 85$ $\%$ ) of the luminosity of a SN Ia emerges at optical wavelengths and this is where they have been best studied to date. Arnett's rule $^{18}$ says the peak luminosity of the $\mathrm{SN}$ is proportional to the mass of nickel-56 produced in the explosion, though in general this is only approximately true $\mathrm{e}^{19,20}$.

The optical light curves of normal SN Ia are relatively homogeneous (and can be standardised as discussed below), with a rise to peak luminosity in $\sim 20$ days, and a slow decline after peak before settling on to an exponential decay phase after $\sim 50$ days (Figure 1). The infrared light curves of SN Ia are characterised by a secondary peak $20-30 \mathrm{~d}$ after maximum light that is not seen at bluer wavelengths, thought to be driven by the recombination of doubly to singly ionised iron ${ }^{21,22}$. SN Ia peak in the ultraviolet (UV) roughly 15-20 d after explosion, slightly before the optical, but with a much lower flux at most epochs $(<10 \%$ of the optical luminosity at peak) due to strong iron-group line blanketing opacity.

Spectral properties of SN Ia: The spectra of SN Ia reveal the elements that are produced in the explosion, their quantities, and their location within the SN ejecta (Figure 2). SN Ia spectra are dominated at early times and maximum light by features from intermediate-mass elements such as calcium, magnesium, silicon, and sulfur, with typical velocities measured from absorption minima of $8000-15000 \mathrm{~km} \mathrm{~s}^{-1}$ around peak and decreasing with time as the photosphere recedes. The earliest spectrum of a SN Ia is SN 2011 fe at just over one day past explosion ${ }^{25}$, and it was remarkably similar to maximum-light spectra of SN Ia, apart from the higher velocities. SN Ia show a spectral sequence in which temperature, ionisation, and line ratios correlate with peak luminosity $^{26,27}$.

After maximum light the spectra begin to be dominated by iron-group elements. The ejecta expand with time, becoming optically thin by $\sim 150$ days past maximum light. SN Ia then enter the "nebular" phase ${ }^{28,29}$ with spectra dominated by forbidden emission lines of singly- and doubly-ionised iron (and other iron-group elements such as cobalt and nickel; Figure 5). At +1000 days the spectrum of SN $2011 \mathrm{fe}$ showed a shift in ionisation to primarily neutral iron ${ }^{30,31}$.

Performing cosmological measurements with SN Ia: As discussed above, SN Ia are best known as extragalactic distance indicators and are essential in precision measurements of the cosmological parameters. These measurements involve the use of empirical corrections to SN Ia light curves to "standardise" their luminosities by correcting for the duration of the light curve (light curve shape or "stretch"), the optical colour at peak brightness ("colour"), as well as a correction for the host galaxy properties of the SN (see Section 2). The original parameterisation of SN Ia light curves was based on the $B$ magnitude decline in the 15 days after maximum light, $\Delta m_{15}(B)$; its correlation with SN Ia luminosity is the Phillips relation ${ }^{8}$. Larger SN Ia samples have refined and extended this relation ${ }^{32}$ leading to the development of modern light-curve fitters to derive SN Ia distances ${ }^{33-35}$. Of particular recent note is the expansion of the wavelength coverage of light-curve models to the near infrared, where SN Ia appear to be more nearly standard ${ }^{36-39}$ (rather than just standardisable; Figure 3) and are less affected by dust extinction.

SN Ia progenitor systems and explosion mechanisms: Understanding how and why stellar systems explode to produce SN Ia is a fundamental astrophysical question and relevant to more precise and accurate SN Ia distances for future cosmological measurements. The research in this area can be divided into two broad categories: studies looking for specific signatures of the companion star to the primary white dwarf and those that try to unveil the explosion mechanism that produces the thermonuclear runaway that unbinds the star. The companion star of SN Ia is thought to be either another degenerate white dwarf or a non-degenerate star such as a main-sequence, giant, or helium star. As such this question is often simplified to asking whether SN Ia arise from single- or double-degenerate systems. However, many more questions remain as to how the explosion begins and proceeds: what kind of material is accreted and how quickly? at what mass does the primary white dwarf explode? does the explosion start as a subsonic deflagration or a supersonic detonation? is the primary white dwarf completely disrupted or is something left behind? what happens to the companion star?

Constraining companion stars: Direct searches for companion stars to normal SN Ia in data taken either before ${ }^{41}$ or relatively soon (centuries) after ${ }^{42-45}$ the explosion have not yielded any detections. Recently three hypervelocity stars discovered in Gaia data have been proposed as older surviving white dwarf companions to SN Ia, arguing for a double-degenerate progenitor system without complete disruption of the donor ${ }^{46}$.

SN Ia light curves within hours to days after explosion (Figure 4) can be used to search for potential shock interaction between the SN ejecta and a companion star or other nearby material, and can also probe properties of the exploding star such as the distribution of nickel-56 and the ejecta density structure. Ground-based data are valuable if there is fast-cadence monitoring of a SN field or if the $\mathrm{SN}$ is discovered early ${ }^{47}$, but the most spectacular early supernova light curves have been observed by the Kepler ${ }^{48-50}$ and now TESS ${ }^{51}$ spacecraft. Early-time "bumps" have clearly been seen in SN 2017cbv and SN 2018oh (Figure 4) but these are not uniquely interpretable as companion shock interaction ${ }^{52-56}$. Observations have generally shown greater variety in SN Ia light curves at early times compared to near or after maximum light ${ }^{57,58}$.

The presence of circumstellar material (CSM), more likely to arise in the single-degenerate scenario, can be investigated 

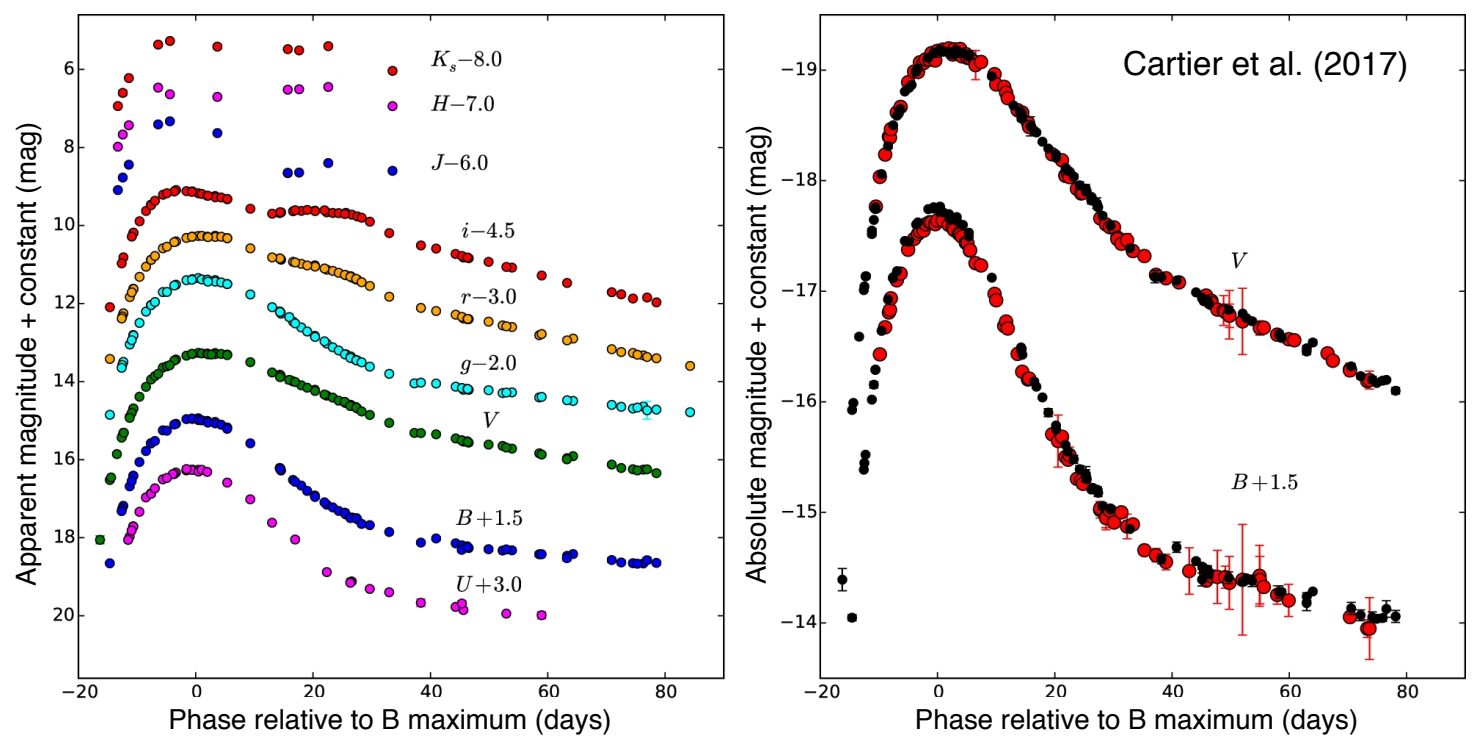

Figure 1 | SN la light curves. Left panel: Optical and near-infrared light curve of the type-la SN 2015F. Right panel: Comparison of the $B$ and $V$ band light curves of SN 2015F (black points) and SN 2004eo (red points) showing the similarity between these two SN la. The error bars displayed are 1- $\sigma$ uncertainties. This figure is adapted from ref. 23.

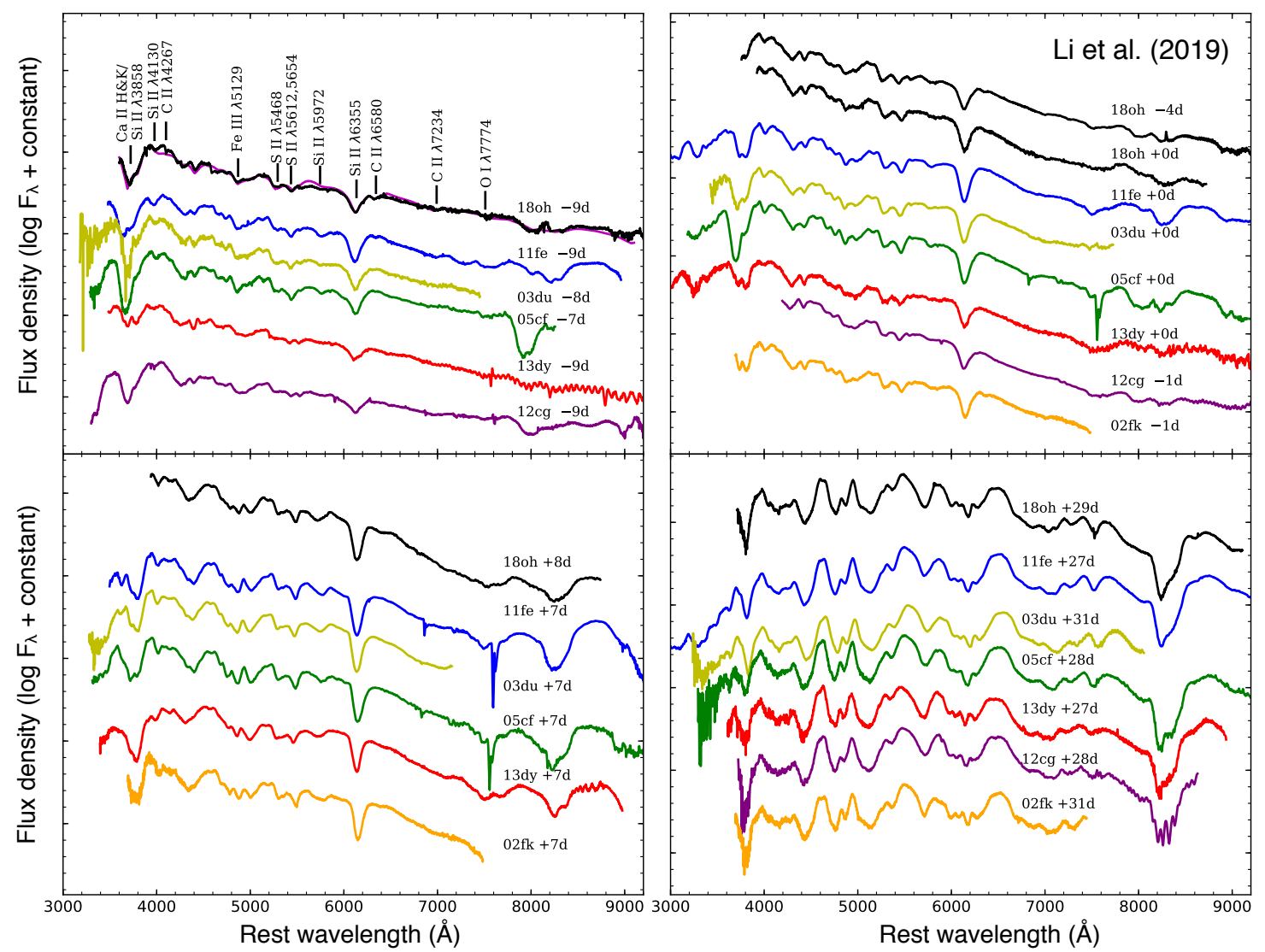

Figure 2 Homogeneous optical spectra of SN la. A sample of SN la is shown at four epochs from -9 days to +1 month after $B$-maximum light. The main contributing features are shown via a model fit (dark magenta) in the upper left panel. This figure is adapted from ref. 24. 


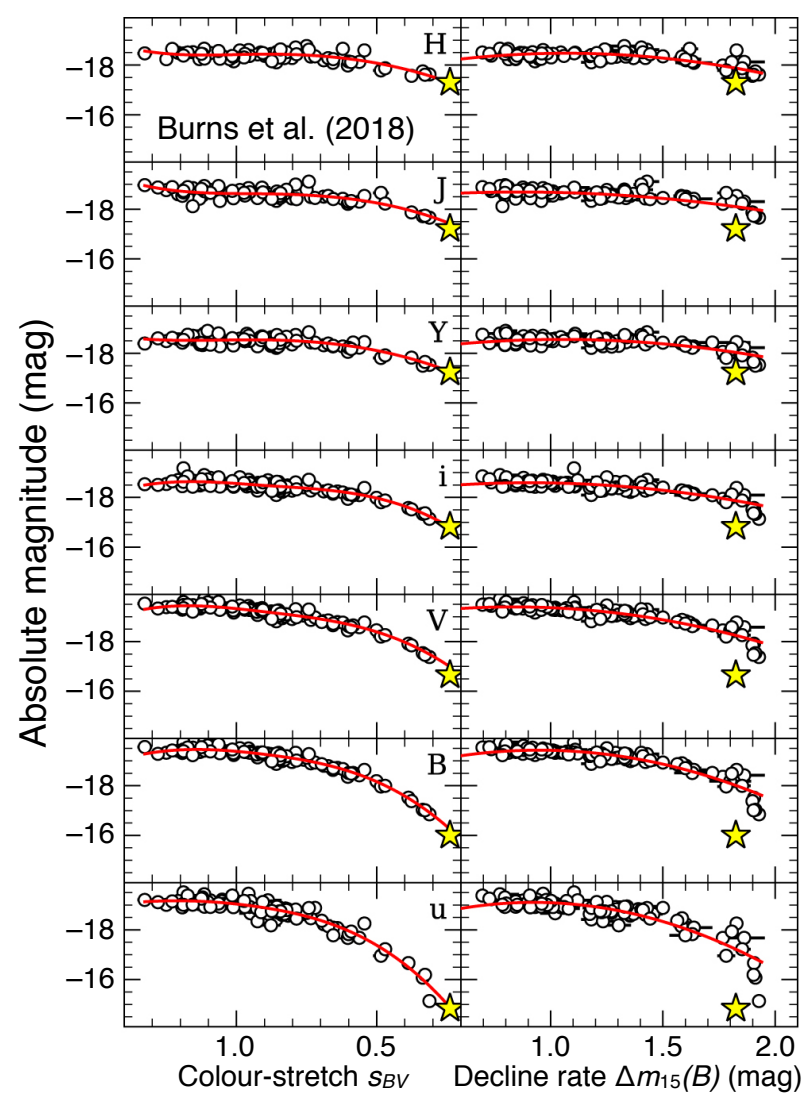

Figure 3 | Light curve shape standardisation of SN la. Modern versions of the Phillips relation ${ }^{8}$ from the Carnegie Supernova Project ${ }^{39}$. The right panels use the original $\Delta m_{15}(B)$ parameterisation, while the left panels use $s_{B V}$, the light-curve colourstretch $^{40}$. Note the tight scatter around the mean relations $(\sigma \lesssim$ $0.15 \mathrm{mag}$, except in $u$ ) and the flattening at longer wavelengths showing that SN la are excellent standard (not just standardisable) candles in the near-infrared. The yellow star is SN $2006 \mathrm{mr}$, illustrating that the colour-stretch more continuously parameterises fastdeclining objects. This figure is adapted from ref. 39.

using radio and X-ray observations. The CSM is expected to be hydrogen-rich in the case of a main-sequence companion star and helium-rich for a helium-star companion. While $\mathrm{X}$ ray and radio emission has been detected for some classes of core-collapse supernovae, searches for X-ray and radio emission of SN Ia have yielded only non-detections. The largest study to date of prompt radio emission $(<1$ year after explosion) of $85 \mathrm{SN}$ Ia resulted in non-detections with upper limits on the pre-explosion mass loss rate, ruling out red giant companions in $>90 \%$ of the sample ${ }^{59}$. X-ray observations have also placed constraining upper limits on the pre-explosion mass loss rate ${ }^{60-62}$.

The CSM of SN Ia can also be studied using the presence of narrow absorption features of $\mathrm{Na} I \mathrm{D}$ and $\mathrm{Ca}$ II that are typically seen in the interstellar medium but can also be present in SN Ia CSM. Blueshifted and time-varying $\mathrm{Na}$ I $\mathrm{D}$ features have been identified in a few SN Ia using high-resolution spectroscopy ${ }^{63}$; a recent example is $\mathrm{SN} 2013 \mathrm{gh}^{64}$. Larger statistical samples have identified excess blueshifted Na I D features in SN Ia, suggesting that there may be outflowing material (consistent with CSM) present in $\sim 20 \%$ of SN Ia ${ }^{65-67}$. High-velocity $\left(>15000 \mathrm{~km} \mathrm{~s}^{-1}\right.$ ) features of calcium (and sometimes silicon) are seen in early to maximum-light spectra of $\sim 80 \% \mathrm{SN} \mathrm{Ia}^{68}$ and are suggested to indicate the presence of CSM or abundance enhancements in the $\mathrm{SN}$ ejecta ${ }^{69}$, but may also result from ionization effects in high velocity material ${ }^{70}$.

A handful of otherwise normal-looking SN Ia, like SN 2002ic ${ }^{71}$ and PTF $11 \mathrm{kx}^{72}$, have shown $H \alpha$ emission, taken as a sign of interaction with hydrogen-rich material. These objects are generically categorised as Ia-CSM ${ }^{73}$. The strength and onset time of the interaction can vary, but objects in this class are typically luminous, slow-declining SN Ia in young environments. Late-time circumstellar interaction is also proposed to explain the ultraviolet emission seen in SN 2015cp nearly two years after the explosion ${ }^{74}$.

Material from a hydrogen- or helium-rich companion star has been predicted to be stripped (or ablated) during the explosion and result in the presence of low-velocity hydrogen- or helium-rich material in the SN ejecta where it can be energized by the radioactive decay and become visible. Searches for this material have been made in many nearby SN Ia using late-time spectra, without detection ${ }^{29,75-84}$. This suggests that either the material is present and is not visible because it is not located cospatially with the radioactive material or that these objects do not have hydrogen- or helium-rich companions.

A unique recent counterexample is ASASSN-18tb, a fastdeclining SN Ia in an early-type host galaxy, which showed nebular-phase $H \alpha$ emission $^{85}$. However TESS early-time observations of ASASSN-18tb did not reveal a companion interaction signature and the nearly constant $H \alpha$ flux may favour a circumstellar interaction power source ${ }^{86}$ even though this supernova is quite different than typical Ia-CSM.

Understanding the explosion mechanism of SN Ia: The properties of the explosion mechanism are difficult to constrain because of the complexities in the model predictions and the intrinsic variations in observed SN Ia properties. Pre-maximum light spectra of SN Ia can show the presence of carbon (and oxygen) features that result from unburned material from the exploding carbon-oxygen white dwarf. The most prominent optical carbon feature is C II $6580 \AA$ and is seen in $>40 \%$ of SN Ia with spectra earlier than 10 days before maximum light ${ }^{87-93}$. Oxygen can be studied via its $7773 \AA$ line, but there is difficulty distinguishing between unburned oxygen and oxygen synthesised in the explosion ${ }^{94}$. The velocity structure of the ejecta can be inferred from spectral timeseries via abundance tomography and generally suggests stratification in $\mathrm{SN} \mathrm{Ia}^{95}$, at odds with pure deflagration (subsonic explosion) models ${ }^{96}$. The high ultraviolet opacity of iron-group elements means that near-UV observations of SN Ia probe the outer layers of the supernova and may provide another avenue to understanding the pre-explosion composition and structure of the white dwarf and the explosion mechanism ${ }^{97,98}$. The abundances of stable iron-group elements (requiring a neutron excess) can be inferred from late- 


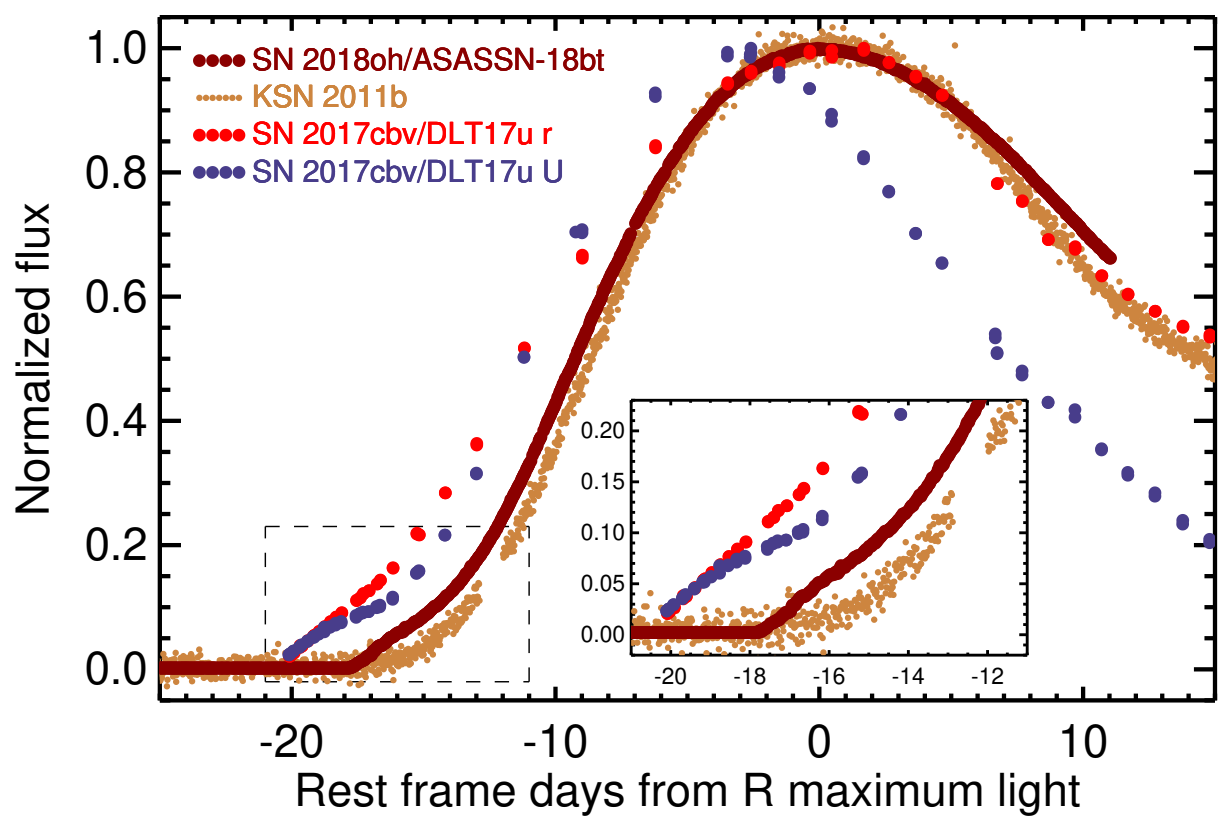

Figure 4 | Early-time SN la light curves. Exquisite Kepler light curves of SN 20180h ${ }^{49,50}$ and KSN $2011 b^{48}$ and early-time groundbased LCOGT light curve of SN $2017 \mathrm{cbv}^{47}$ showing the diverse behaviour of SN la in the days after explosion. The inset shows a zoom on the dashed box. Deviations from a smooth early rise have been interpreted as shock interaction with a non-degenerate companion, but they may alternatively result from radioactivity in the outer $\mathrm{SN}$ ejecta.

time spectra ${ }^{99}$; these are sensitive to the density of the burning material and can help distinguish between Chandrasekhar mass $\left(M_{\mathrm{Ch}}\right)$ and sub-Chandra explosions ${ }^{100}$.

Polarisation measurements of SN provide details on the geometry of the ejecta and the extent of any asymmetries. Continuum polarisation is found to be small in SN Ia, suggesting deviations of $<10 \%$ from spherical symmetry. However, line polarisation has been found to be common in $\mathrm{SN}$ Ia, with significant polarisation observed across the $\mathrm{Si}$ II and $\mathrm{Ca}$ II features that may suggest a separate line-forming region or an asymmetric distribution for these elements (at least at early times) ${ }^{102-104}$. Potential asymmetries in the ejecta distribution (and hence the explosion) can also be studied by looking at late-time spectra, where the outer layers have become transparent and the core of the ejecta becomes visible. Shifts of up to $\sim 3000 \mathrm{~km} \mathrm{~s}^{-1}$ (both to the blue and to the red) in iron and nickel forbidden emission lines have been identified in spectra at $\sim 200$ days past maximum, indicating relatively large asymmetries in the inner Fe-rich ejecta, and these seem to be correlated with early-time properties (Figure 5), perhaps suggesting an orientation effect ${ }^{99,101}$. Approximately $15 \%$ of SN Ia show signatures of double-peaked nebular emission lines separated by $\sim 5000 \mathrm{~km} \mathrm{~s}^{-1}$, and the fraction rises for subluminous $\mathrm{SN} \mathrm{Ia}{ }^{105,106}$; this may result from two explosion sites in a white dwarf collision model.

\section{SN la and their environments}

SN Ia have been observed to occur in every type of galactic environment, across galaxy types, stellar masses, metallicities and ages, from the lowest mass dwarf galaxies to the most massive ellipticals. This simple observation of ubiquity has significant implications for understanding the $\mathrm{SN}$ Ia progenitor system and explosion physics: a SN Ia explosion must be able to result from a progenitor with a wide range of stellar ages, from young to very old systems. Detailed observations of SN Ia environments can provide further clues.

SN Ia rates: The specific SN Ia rate (the SN Ia rate per unit stellar mass) is significantly higher in star-forming later-type galaxies than in early-type systems ${ }^{107,108}$. Similar higher specific rates are observed in bluer host galaxies compared to red host galaxies ${ }^{108}$, lower mass galaxies compared to higher mass galaxies ${ }^{109,110}$, and in host galaxies with high specific starformation rates ${ }^{109,111}$, i.e. the star formation rate (SFR) per unit stellar mass. The logical inference is that SN Ia are more common in younger progenitor systems compared to older progenitor systems, with a "delay-time distribution" $\left(\mathrm{DTD}^{1}\right)$ that decreases sharply with progenitor age. More detailed analyses have shown that these observations are a natural consequence of power-law DTDs ${ }^{112,113}$.

These observations are consistent with the observed redshift evolution in the volumetric SN Ia rate (the rate of SN Ia per co-moving volume). The volumetric SN Ia rate increases with increasing redshift, and by combining volumetric rate measurements from different surveys across a range of redshifts, sev-

\footnotetext{
${ }^{1}$ The SN Ia DTD describes the SN Ia rate as a function of time following an instantaneous burst of star formation. Thus it describes the likelihood of a SN Ia explosion occurring as a function of the progenitor age.
} 

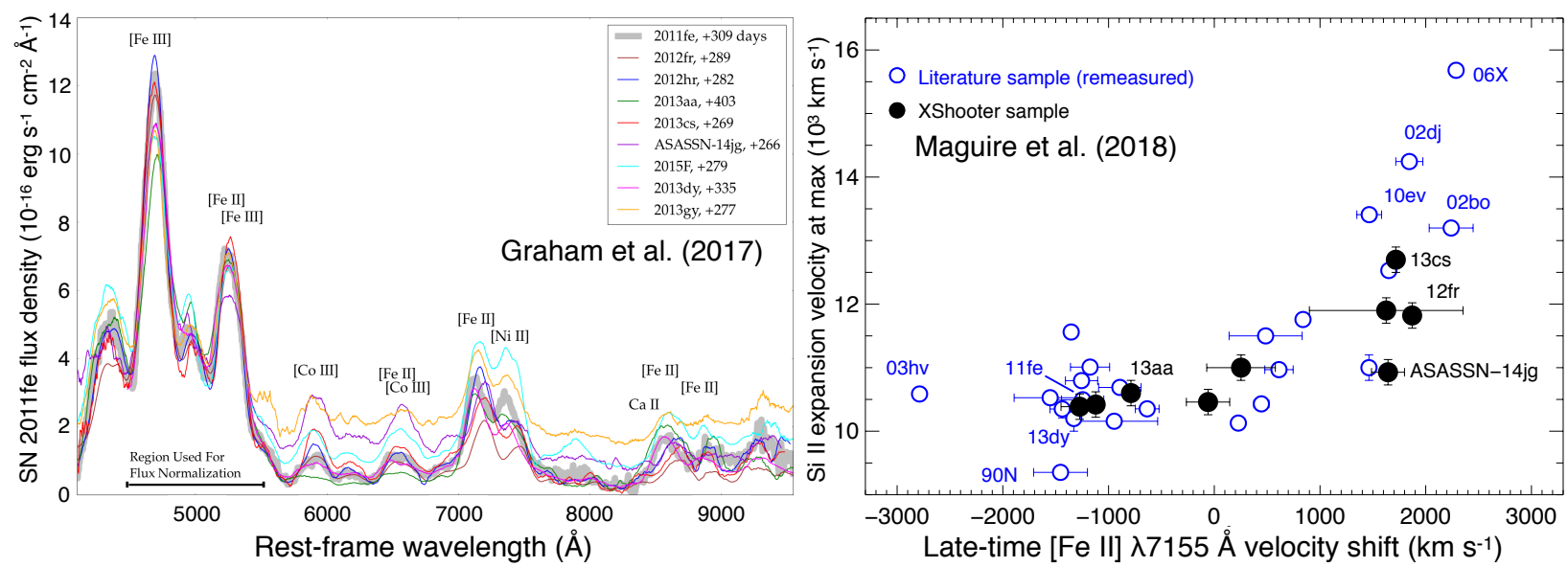

Figure 5 | SN la nebular spectroscopy and line shifts. Nebular spectra of SN la (left panel) and velocity of the Si II absorption feature measured at maximum light versus the velocity shift of the Fe II emission line in late-time spectra of a sample of SN la (right panel). The error bars displayed are 1- $\sigma$ uncertainties. A trend is seen where SN la with redshifted Fe II features have higher maximum light Si II velocities. The origin of this trend is debated but may be related to asymmetries in the explosion mechanism and ejecta ${ }^{101}$. This figure is adapted from refs. 29 (left panel) and 99 (right panel).

eral studies ${ }^{114-116}$ have demonstrated that this redshift evolution in the cosmic SN Ia rate is consistent with a power-law DTD $\sim t^{-1}$, favouring double-degenerate progenitor systems.

Environmental dependence of SN Ia properties: The observed variation in the rate of SN Ia with the age of the progenitor stellar population extends to some observed properties of SN Ia events. A key observable affecting the utility of SN Ia as cosmological probes is the light-curve-width/luminosity relationship (Section 1): brighter SN Ia have light curves that evolve more slowly. It has been known for more than twenty years that this light-curve width correlates with host galaxy properties $^{109,117-119}$, with brighter, slower SN Ia being hosted by younger, less massive, and more strongly star-forming galaxies. This observation is, or should be, potentially alarming: the fundamental standardising variable used in SN Ia cosmology depends on the age of the SN Ia progenitor system. This implies that the distribution of this parameter in SN Ia populations should evolve with redshift, with a predicted shift to SN Ia with brighter, slower light curves at high redshift ${ }^{120}$. Such variations in SN Ia properties also have implications for progenitor systems and explosion scenarios ${ }^{121}$.

Impact on SN Ia distances and cosmology: These relationships between SN photometric properties and host galaxy properties demand that if SN Ia are to be good standardisable candles over cosmic time, the calibrating relationships between SN Ia luminosity and light-curve shape must be invariant with progenitor age (or $\mathrm{SN}$ environment). In current samples, this appears to be the case, at least to the level that it can currently be measured. The relationships between luminosity and light-curve shape do not show significant dependence on host galaxy properties.

More subtle trends in SN Ia properties as a function of environment have also been detected: the standardised distance estimated from SN Ia has a small dependence on the proper- ties of the SN host galaxies. This was originally observed to occur as a function of the host galaxy stellar mass ${ }^{122-124}$, with brighter SN Ia (after light-curve width and colour corrections) occurring in higher-mass galaxies - this is in the opposite sense to the far larger trend between uncorrected SN Ia luminosities and host properties. Galaxy stellar mass is unlikely to be the fundamental variable or root cause of this relationship - galaxy stellar mass correlates with many other physical quantities, such as metallicity (gas-phase and stellar), stellar age, and galaxy dust content. The trend is also seen with many of these other global host galaxy properties ${ }^{125}$, but global properties alone seem unlikely to be able to resolve the physical cause of the variation, given the covariance between the different variables. Selection effects, sample data quality, and the choice of light curve standardisation may also play a role ${ }^{126,127}$.

Measurements of local galaxy properties at the SN position hold promise to clarify the picture (Figure 6). SN Ia Hubble residuals show a correlation with local specific star formation rate ${ }^{128-130}$, measured either with nearby nebular $H \alpha$ emission, rest-frame near-UV flux, or galaxy SED fitting to optical colours, though there remains some disagreement as to whether corrections based on these local measures should be used in preference to those based on global host properties. Most of this work has focused on SN Ia in the local universe, where such studies are simpler to perform as the $\mathrm{SN}$ Ia host galaxies are better resolved. However, a similar trend is also seen at moderate redshift using local photometry ${ }^{131}$ and in high-redshift samples using rest-frame UV photometry measured in $3 \mathrm{kpc}$ apertures from deep imaging stacks ${ }^{132}$. SN Ia in locally star-forming regions may also have a lower dispersion than the rest ${ }^{128,133}$.

The interpretation of these results is unclear. There is a prediction from empirical galaxy models that low-mass galaxies should be expected to contain a more homogeneous popu- 

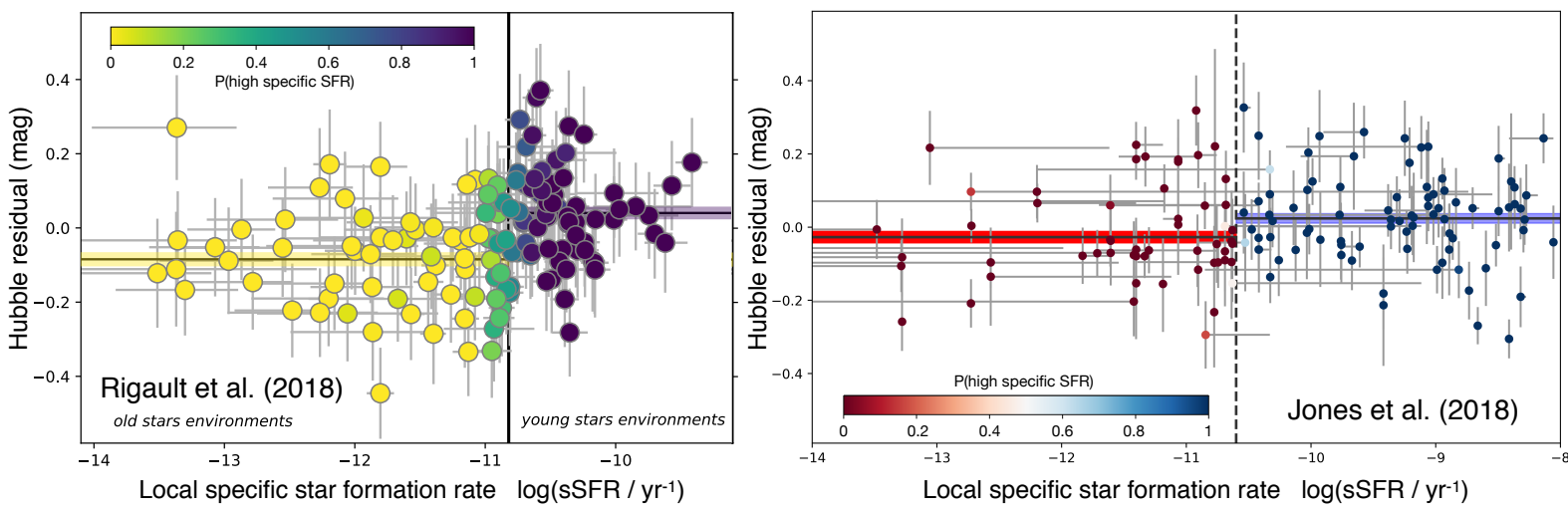

Figure 6 | The effect of environment on SN la standardisation. SN la Hubble diagram residuals as a function of the local specific star-formation rate (sSFR), the star formation rate per unit stellar mass. Left: The sSFR is measured using $\mathrm{H} \alpha$ emission measured in a $1 \mathrm{kpc}$ projected average radius; Right: The sSFR is measured using SED fitting to broad-band optical data in $1.5 \mathrm{kpc}$ projected apertures. The colours show the probability for a SN to have a younger environment. The two horizontal bands give the weighted average of the Hubble residuals per sSFR group. The width of each band represents the corresponding error on the mean, and their offset illustrates the Hubble residual offset between the two sSFR groups. The error bars displayed are 1- $\sigma$ uncertainties. This figure is adapted from refs. 128 (left panel) and 129 (right panel).

lation of young SN Ia progenitors across all redshift ranges ${ }^{134}$. Assuming these lower-mass galaxies are also more strongly starforming, this is consistent with observations that SN Ia in starforming galaxies present a more homogeneous population. Selecting these events in cosmological studies may therefore provide access to a SN Ia sample with a narrow range in progenitor ages, therefore removing the challenge of using corrections in cosmological analyses based on host environment, and perhaps reducing potential astrophysical systematic effects when using SN Ia in cosmology.

\section{The Thermonuclear Supernova Zoo}

The Phillips relation ${ }^{8}$ defines a one-parameter family of $\mathrm{SN}$ Ia, seen also as a spectral sequence ${ }^{26}$. The slow-declining, hot, luminous end is marked by 91T-like or 99aa-like objects, showing prominent Fe III features with weak Si II in maximumlight spectra. 99aa-like SN Ia also show strong Ca II absorption that is much weaker in 91T-like objects ${ }^{135,136}$. The fast-declining, cool, subluminous 91bg-like SN Ia are most often found in old stellar populations ${ }^{137}$ (i.e., passive host galaxies) and are sometimes claimed to be a separate population from more normal SN Ia, with few "transitional" objects in between ${ }^{23,138}$. However, this may only be pointing to a shortcoming of the $\Delta m_{15}$ parameterisation ${ }^{8}$; using colour ${ }^{139}$ or colour-stretch ${ }^{39,40}$ suggests a more continuous distribution with other SN Ia (Figure 3). Off the Phillips relation is the realm of "peculiar" thermonuclear supernovae. The same luminosity/decline-rate parameter space has been used to distinguish these objects ${ }^{135}$ (Figure 7).

Several groups of white dwarf supernovae show low ejecta velocities, below the typical $10,000 \mathrm{~km} \mathrm{~s}^{-1} \mathrm{Si}$ II velocity seen in normal SN Ia around maximum light. The most numerous of these are type Iax supernovae ${ }^{141,142}$ (SN Iax) with SN 2002cx as the prototype ${ }^{143,144}$. Though typically found in star-forming environments, SN Iax are thought to be white-dwarf supernovae because of their spectral similarity to normal SN Ia at early times, including the dominance of iron-group elements (like the Co II infrared lines ${ }^{145,146}$; Figure 8 ) as well as their radioactively powered light curves ${ }^{147}$. Near maximum light SN Iax have low photospheric velocities (from 7000 down to $2000 \mathrm{~km} \mathrm{~s}^{-1}$ ) and typically low luminosity $\left(-19 \lesssim M_{V} \lesssim-13\right.$ ) compared to normal SN Ia, and show more overall diversity ${ }^{142}$. At late times SN Iax differ from all other supernovae in never becoming fully "nebular" in their spectra, with the marked presence of low velocity $\left(<2000 \mathrm{~km} \mathrm{~s}^{-1}\right)$ permitted iron lines. The SN Iax $2012 Z$ is unique among all thermonuclear supernovae because of the detection of its luminous progenitor system in prediscovery Hubble Space Telescope images ${ }^{148}$, interpreted as a helium-star donor to the exploding white dwarf. A leading model for SN Iax is the pure-deflagration explosion of a Chandrasekhar-mass white dwarf in a helium-accreting singledegenerate system ${ }^{142}$. It is possible this explosion does not completely disrupt the white dwarf, leaving a bound remnant ${ }^{149}$. Surviving examples of such incomplete explosions may have been discovered in our Galaxy as fast-moving white dwarfs with unusual abundances ${ }^{150,151}$.

Perhaps related to the class of SN Iax are 02es-like ${ }^{135,152}$ SN which also have low luminosity and low ejecta velocity, but "cool" spectra compared to the "hot" SN Iax. Those properties and the preference for 02es-likes for old stellar environments are similar to $91 \mathrm{bg}$-like SN Ia, but 02es-likes do not have fast lightcurves. The 02es-like iPTF 14atg showed evidence of an earlytime "blue bump" that may be consistent with shock interaction of the supernova ejecta with a companion $\operatorname{star}^{153}$. SN 2010lp was an 02es-like object that showed nebular oxygen emission ${ }^{154}$ and a similar feature was observed in iPTF 14 atg $^{155}$; such emission has not been seen in any other thermonuclear SN. 


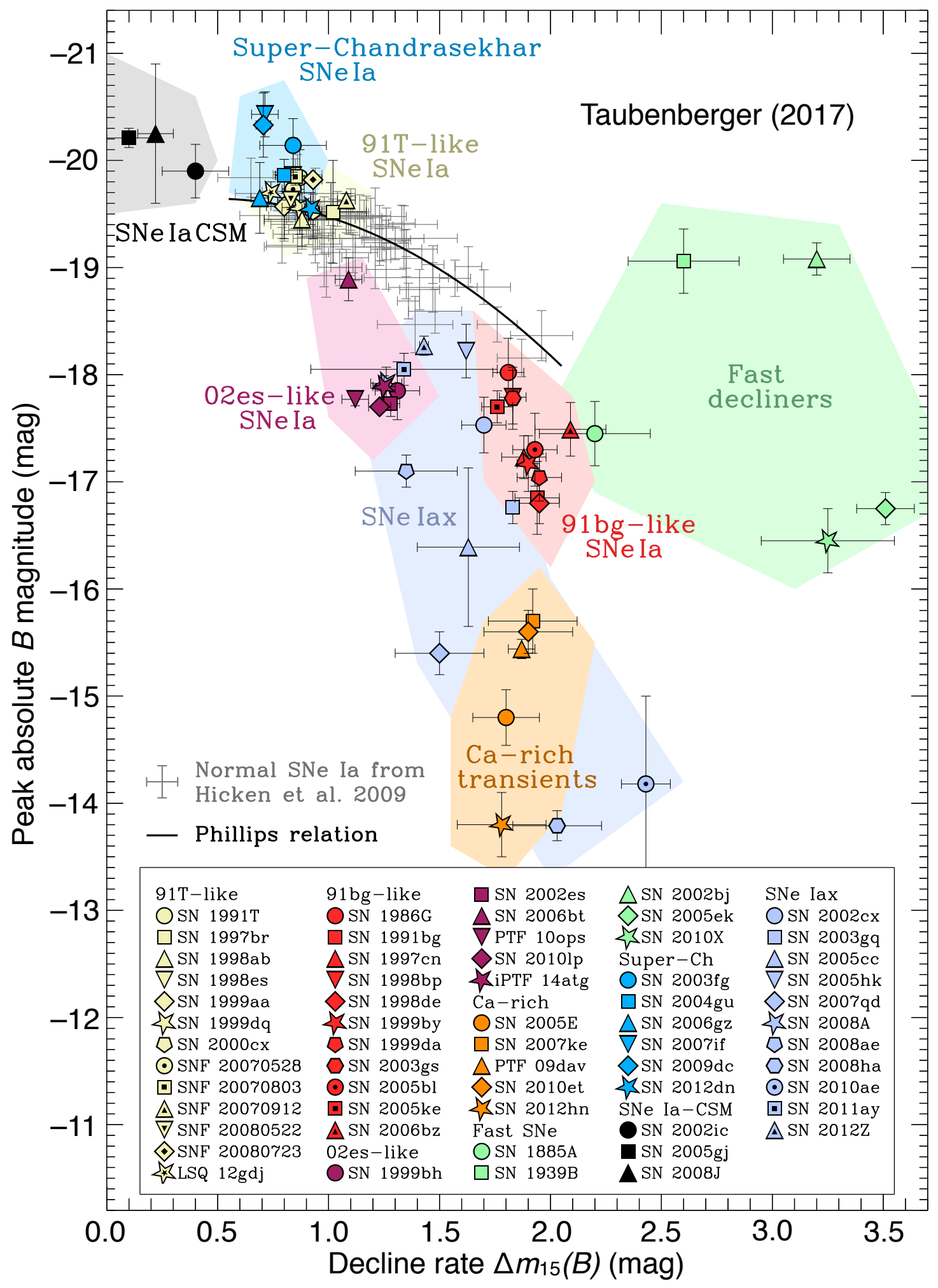

Figure 7 | The thermonuclear supernova zoo. Luminosity versus light-curve decline rate of normal SN la, extreme SN la, and the wide variety of peculiar white dwarf supernovae. The error bars displayed are 1- $\sigma$ uncertainties. This figure is adapted from ref. 135. 


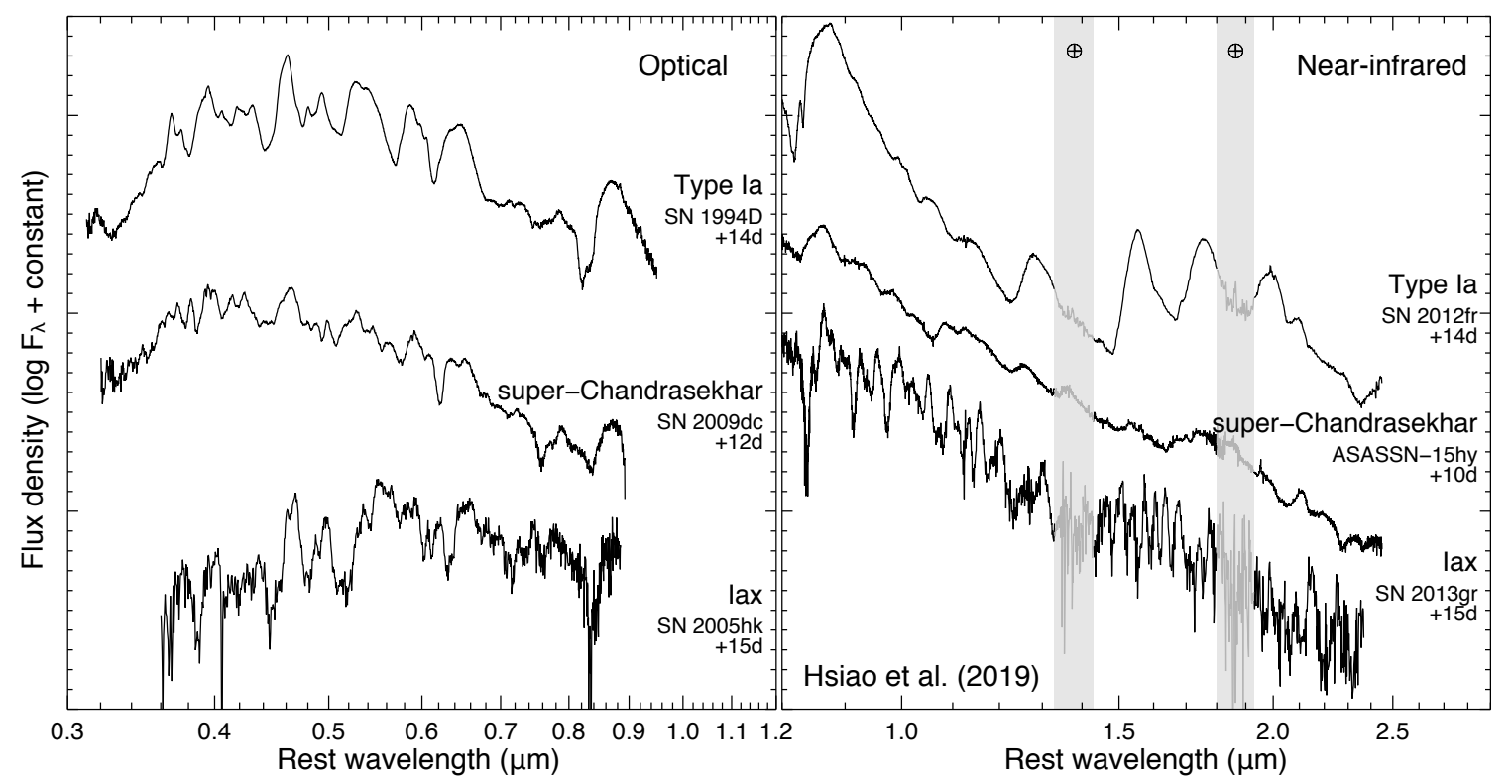

Figure 8 | Optical and near-infrared spectroscopy of thermonuclear supernovae. Near-infrared spectroscopy (right) illustrates the differences between subclasses of thermonuclear supernovae more clearly than optical spectra (left). This figure is adapted from ref. 140.

"Super-Chandrasekhar" SN Ia have optical spectra similar to normal SN Ia, though with strong carbon features near maximum light and more distinct near-infrared spectra (Figure 8). They have relatively high luminosity, slow light curves, and low ejecta velocities. Taken together these properties imply a high ejecta mass, exceeding $M_{\mathrm{Ch}}$, thus explaining the "superChandra" moniker ${ }^{135,156-159}$. An observationally-based name for this class may be preferable to a model-dependent one ${ }^{160}$, but these objects and their progenitors regardless raise fundamental astrophysics questions. One clue to their nature may come from a preference for low-metallicity environments ${ }^{161}$.

Another class of objects for which environments may be the key to understanding is "calcium-rich" supernovae. Spectroscopically similar to type-Ib supernovae, with prominent helium features at maximum light, these low-luminosity explosions occur far from any star formation, and indeed often far from their host galaxies with almost no underlying stellar light ${ }^{162-165}$. In their nebular spectra these objects are dominated by strong [Ca II] emission, giving them their name. A proposed origin is a long delay-time thermonuclear explosion of a white dwarf in a binary system that was dynamically ejected from its host ${ }^{166,167}$, but multiple progenitor scenarios may be required ${ }^{168,169}$.

The thermonuclear supernova zoo also contains unusual objects which have not (yet) been easily grouped into classes, e.g., SN 2000cx and its twin SN 2013bh ${ }^{170-172}$; "fast and faint" objects like SN 2005ek ${ }^{173}$, PTF09dav ${ }^{174}$, SN 2010X ${ }^{175}$, and even SN $1885 \mathrm{~A}^{176}$; the slow and faint PTF10ops ${ }^{177}$; and the fast and not-so-faint SN 1939B ${ }^{178}$, and SN 2002bj ${ }^{179}$. Unique objects continue to be discovered, like the high-velocity SN 2019ein ${ }^{180}$. A number of peculiar thermonuclear transients have shown evidence for detonation of a helium shell ${ }^{181,182}$ and may imply a diversity in total mass and shell mass for exploding white dwarfs ${ }^{56}$. Though the zoo is stocked with a broad variety of thermonuclear SN, volumetric rates of these species "in the wild" can vary widely. The luminous peculiar objects, like superChandra or Ia-CSM, are intrinsically rare and not more than a few percent of the SN Ia rate ${ }^{3}$. Subluminous peculiar objects are more common ${ }^{141,183,184}$, but it is nonetheless likely that normal SN Ia that lie on the Phillips relation still comprise the most numerous class of thermonuclear supernovae. This remarkable fact needs an explanation.

In this review we have tried to highlight recent advances in our observational understanding of thermonuclear supernovae. Even with this limited aim our review is incomplete, and moreover, we have not been able to sufficiently discuss important progress from theory and computation on models of white dwarf supernova progenitor systems and explosions. These shortcomings testify to the vibrancy of the field. We should make special note that the bulk of the observational progress described here is predicated on the increasing number of bright or nearby supernovae (and their earlier discovery) from a number of surveys like ASASSN ${ }^{185}$, ATLAS $^{186}$, CRTS $^{187}$, DLT40 $^{188}$, Gaia $^{189}$, LOSS $^{190}$, LSQ $^{191}$, MASTER $^{192}$, OGLE $^{193}$, Pan-STARRS $^{194}$, PTF $/$ iPTF $^{195}$, PTSS $^{196}, \mathrm{ZTF}^{197}$, among others, and the continued work of amateur astronomers. Upcoming surveys like $\operatorname{LSST}^{198}$ will provide large samples of more distant supernovae, including rare objects, and will allow nearby supernovae to be systematically observed to late times as they fade. Such samples will help develop a deeper physical understanding of thermonuclear supernova progenitors and explosions and can be used to improve distances from SN Ia and measurements of cosmological parameters. 
1. Minkowski, R. Spectra of Supernovae. Publ. Astron. Soc. Pac. 53, 224 (1941).

2. Filippenko, A. V. Optical Spectra of Supernovae. Annu. Rev. Astron. Astrophys. 35, 309-355 (1997).

3. Gal-Yam, A. Observational and physical classification of supernovae. In Alsabti, A. W. \& Murdin, P. (eds.) Handbook of Supernovae, 1-43 (Springer International Publishing, Cham, 2017). Preprint at http://arxiv.org/abs/1611.09353.

4. Bloom, J. S. et al. A Compact Degenerate Primary-star Progenitor of SN 2011fe. Astrophys. J. 744, L17 (2012).

5. Churazov, E. et al. Cobalt-56 $\gamma$-ray emission lines from the type Ia supernova 2014J. Nature 512, 406-408 (2014).

6. Piro, A. L. \& Nakar, E. What can we Learn from the Rising Light Curves of Radioactively Powered Supernovae? Astrophys. J. 769, 67 (2013).

7. Diehl, R. et al. Early ${ }^{56} \mathrm{Ni}$ decay gamma rays from SN2014J suggest an unusual explosion. Science 345, 1162-1165 (2014).

8. Phillips, M. M. The absolute magnitudes of Type Ia supernovae. Astrophys. J. 413, L105-L108 (1993).

9. Riess, A. G. et al. Observational Evidence from Supernovae for an Accelerating Universe and a Cosmological Constant. Astron. J. 116 1009-1038 (1998)

10. Perlmutter, S. et al. Measurements of $\Omega$ and $\Lambda$ from 42 High-Redshift Supernovae. Astrophys. J. 517, 565-586 (1999).

11. Riess, A. G. et al. A $2.4 \%$ Determination of the Local Value of the Hubble Constant. Astrophys. J. 826, 56 (2016).

12. Nomoto, K., Kobayashi, C. \& Tominaga, N. Nucleosynthesis in Stars and the Chemical Enrichment of Galaxies. Annu. Rev. Astron. Astrophys. 51, 457-509 (2013).

13. Hillebrandt, W., Kromer, M., Röpke, F. K. \& Ruiter, A. J. Towards an understanding of Type Ia supernovae from a synthesis of theory and observations. Frontiers of Physics 8, 116-143 (2013).

14. Alsabti, A. W. \& Murdin, P. Handbook of Supernovae (Springer International Publishing, Cham, 2017)

15. Branch, D. \& Wheeler, J. C. Supernova Explosions (Springer, Berlin, 2017).

16. Pankey, T., Jr. Possible Thermonuclear Activities in Natural Terrestrial Minerals. Ph.D. thesis, Howard University (1962).

17. Colgate, S. A. \& McKee, C. Early Supernova Luminosity. Astrophys. J. 157, 623 (1969).

18. Arnett, W. D. Type I supernovae. I - Analytic solutions for the early part of the light curve. Astrophys. J. 253, 785-797 (1982).

19. Katz, B., Kushnir, D. \& Dong, S. An exact integral relation between the Ni56 mass and the bolometric light curve of a type Ia supernova. Preprint at http://arxiv.org/abs/1301.6766 (2013).

20. Khatami, D. K. \& Kasen, D. N. Physics of Luminous Transient Light Curves: A New Relation between Peak Time and Luminosity. Astrophys. J. 878, 56 (2019).

21. Kasen, D. Secondary Maximum in the Near-Infrared Light Curves of Type Ia Supernovae. Astrophys. J. 649, 939-953 (2006).

22. Kromer, M. et al. Double-detonation Sub-Chandrasekhar Supernovae: Synthetic Observables for Minimum Helium Shell Mass Models. As trophys. J. 719, 1067-1082 (2010).

23. Cartier, R. et al. Early observations of the nearby Type Ia supernova SN 2015F. Mon. Not. R. Astron. Soc. 464, 4476-4494 (2017).

24. Li, W. et al. Photometric and Spectroscopic Properties of Type Ia Supernova 2018oh with Early Excess Emission from the Kepler 2 Observations. Astrophys. J. 870, 12 (2019).

25. Nugent, P. E. et al. Supernova SN $2011 \mathrm{fe}$ from an exploding carbonoxygen white dwarf star. Nature 480, 344-347 (2011).

26. Nugent, P., Phillips, M., Baron, E., Branch, D. \& Hauschildt, P. Evidence for a Spectroscopic Sequence among Type 1a Supernovae. Astrophys. J. 455, L147+ (1995).
27. Hachinger, S., Mazzali, P. A., Tanaka, M., Hillebrandt, W. \& Benetti, $\mathrm{S}$. Spectral luminosity indicators in Type Ia supernovae. Understanding the (SilI) line-strength ratio and beyond. Mon. Not. R. Astron. Soc. 389, 1087-1096 (2008).

28. Silverman, J. M., Ganeshalingam, M. \& Filippenko, A. V. Berkeley Supernova Ia Program - V. Late-time spectra of Type Ia Supernovae. Mon. Not. R. Astron. Soc. 430, 1030-1041 (2013).

29. Graham, M. L. et al. Nebular-phase spectra of nearby Type Ia Supernovae. Mon. Not. R. Astron. Soc. 472, 3437-3454 (2017).

30. Taubenberger, S. et al. Spectroscopy of the Type Ia supernova $2011 \mathrm{fe}$ past 1000 d. Mon. Not. R. Astron. Soc. 448, L48-L52 (2015).

31. Fransson, C. \& Jerkstrand, A. Reconciling the Infrared Catastrophe and Observations of SN 2011fe. Astrophys. J. 814, L2 (2015).

32. Hamuy, M. et al. The Absolute Luminosities of the Calan/Tololo Type IA Supernovae. Astron. J. 112, 2391 (1996)

33. Guy, J. et al. SALT2: using distant supernovae to improve the use of type Ia supernovae as distance indicators. Astron. Astrophys. 466, 11-21 (2007).

34. Jha, S., Riess, A. G. \& Kirshner, R. P. Improved Distances to Type Ia Supernovae with Multicolor Light-Curve Shapes: MLCS2k2. Astrophys. J. 659, 122-148 (2007).

35. Burns, C. R. et al. The Carnegie Supernova Project: Light-curve Fitting with SNooPy. Astron. J. 141, 19 (2011).

36. Krisciunas, K., Phillips, M. M. \& Suntzeff, N. B. Hubble Diagrams of Type Ia Supernovae in the Near-Infrared. Astrophys. J. 602, L81-L84 (2004).

37. Barone-Nugent, R. L. et al. Near-infrared observations of Type Ia supernovae: the best known standard candle for cosmology. Mon. Not. R. Astron. Soc. 425, 1007-1012 (2012).

38. Dhawan, S., Jha, S. W. \& Leibundgut, B. Measuring the Hubble constant with Type Ia supernovae as near-infrared standard candles. Astron. Astrophys. 609, A72 (2018).

39. Burns, C. R. et al. The Carnegie Supernova Project: Absolute Calibration and the Hubble Constant. Astrophys. J. 869, 56 (2018).

40. Burns, C. R. et al. The Carnegie Supernova Project: Intrinsic Colors of Type Ia Supernovae. Astrophys. J. 789, 32 (2014)

41. Li, W. et al. Exclusion of a luminous red giant as a companion star to the progenitor of supernova SN 2011fe. Nature 480, 348-350 (2011).

42. Schaefer, B. E. \& Pagnotta, A. An absence of ex-companion stars in the type Ia supernova remnant SNR 0509-67.5. Nature 481, 164-166 (2012).

43. Kerzendorf, W. E. et al. A High-resolution Spectroscopic Search for the Remaining Donor for Tycho's Supernova. Astrophys. J. 774, 99 (2013).

44. Kerzendorf, W. E. et al. A search for a surviving companion in SN 1006. Mon. Not. R. Astron. Soc. 479, 192-199 (2018).

45. Ruiz-Lapuente, P. et al. No Surviving Companion in Kepler's Supernova. Astrophys. J. 862, 124 (2018).

46. Shen, K. J. et al. Three Hypervelocity White Dwarfs in Gaia DR2: Evidence for Dynamically Driven Double-degenerate Double-detonation Type Ia Supernovae. Astrophys. J. 865, 15 (2018)

47. Hosseinzadeh, G. et al. Early Blue Excess from the Type Ia Supernova $2017 \mathrm{cbv}$ and Implications for Its Progenitor. Astrophys. J. 845, L11 (2017).

48. Olling, R. P. et al. No signature of ejecta interaction with a stellar companion in three type Ia supernovae. Nature 521, 332-335 (2015).

49. Dimitriadis, G. et al. K2 Observations of SN 2018oh Reveal a Twocomponent Rising Light Curve for a Type Ia Supernova. Astrophys. J. 870, L1 (2019).

50. Shappee, B. J. et al. Seeing Double: ASASSN-18bt Exhibits a Twocomponent Rise in the Early-time K2 Light Curve. Astrophys. J. 870, 13 (2019).

51. Fausnaugh, M. M. et al. Early Time Light Curves of 18 Bright Type Ia Supernovae Observed with TESS. Preprint at http://arxiv.org/abs/1904.02171 (2019). 
52. Piro, A. L. \& Nakar, E. What can we Learn from the Rising Ligh Curves of Radioactively Powered Supernovae? Astrophys. J. 769, 67 (2013).

53. Maeda, K., Jiang, J.-a., Shigeyama, T. \& Doi, M. Type Ia Supernovae in the First Few Days: Signatures of Helium Detonation versus Interaction. Astrophys. J. 861, 78 (2018).

54. Magee, M. R., Sim, S. A., Kotak, R. \& Kerzendorf, W. E. Modelling the early time behaviour of type Ia supernovae: effects of the ${ }^{56} \mathrm{Ni}$ distribution. Astron. Astrophys. 614, A115 (2018).

55. Stritzinger, M. D. et al. Red versus Blue: Early Observations of Thermonuclear Supernovae Reveal Two Distinct Populations? Astrophys. J. 864, L35 (2018).

56. Polin, A., Nugent, P. \& Kasen, D. Observational Predictions for SubChandrasekhar Mass Explosions: Further Evidence for Multiple Progenitor Systems for Type Ia Supernovae. Astrophys. J. 873, 84 (2019)

57. Hayden, B. T. et al. The Rise and Fall of Type Ia Supernova Ligh Curves in the SDSS-II Supernova Survey. Astrophys. J. 712, 350-366 (2010).

58. Firth, R. E. et al. The rising light curves of Type Ia supernovae Mon. Not. R. Astron. Soc. 446, 3895-3910 (2015).

59. Chomiuk, L. et al. A Deep Search for Prompt Radio Emission from Thermonuclear Supernovae with the Very Large Array. Astrophys. $J$. 821, 119 (2016).

60. Russell, B. R. \& Immler, S. Swift X-Ray Upper Limits on Type Ia Supernova Environments. Astrophys. J. 748, L29 (2012).

61. Horesh, A. et al. Early Radio and X-Ray Observations of the Youngest nearby Type Ia Supernova PTF 11kly (SN 2011fe). Astrophys. J. 746, 21 (2012).

62. Margutti, R. et al. No X-Rays from the Very Nearby Type Ia SN 2014J Constraints on Its Environment. Astrophys. J. 790, 52 (2014).

63. Patat, F. et al. Detection of Circumstellar Material in a Normal Type Ia Supernova. Science 317, 924- (2007).

64. Ferretti, R. et al. Time-varying sodium absorption in the Type Ia supernova 2013gh. Astron. Astrophys. 592, A40 (2016).

65. Sternberg, A. et al. Circumstellar Material in Type Ia Supernovae via Sodium Absorption Features. Science 333, 856- (2011).

66. Maguire, K. et al. A statistical analysis of circumstellar material in Type Ia supernovae. Mon. Not. R. Astron. Soc. 436, 222-240 (2013).

67. Phillips, M. M. et al. On the Source of the Dust Extinction in Type Ia Supernovae and the Discovery of Anomalously Strong Na I Absorption. Astrophys. J. 779, 38 (2013).

68. Mazzali, P. A. et al. High-Velocity Features: A Ubiquitous Property of Type Ia Supernovae. Astrophys. J. 623, L37-L40 (2005)

69. Zhao, X. et al. The Silicon and Calcium High-velocity Features in Type Ia Supernovae from Early to Maximum Phases. Astrophys. J. Suppl. Ser. 220, 20 (2015)

70. Blondin, S., Dessart, L., Hillier, D. J. \& Khokhlov, A. M. Onedimensional delayed-detonation models of Type Ia supernovae: confrontation to observations at bolometric maximum. Mon. Not. R. Astron. Soc. 429, 2127-2142 (2013).

71. Hamuy, M. et al. An asymptotic-giant-branch star in the progenitor system of a type Ia supernova. Nature 424, 651-654 (2003).

72. Dilday, B. et al. PTF 11kx: A Type Ia Supernova with a Symbiotic Nova Progenitor. Science 337, 942 (2012).

73. Silverman, J. M. et al. Type Ia Supernovae Strongly Interacting with Their Circumstellar Medium. Astrophys. J. Suppl. Ser. 207, 3 (2013).

74. Graham, M. L. et al. Delayed Circumstellar Interaction for Type Ia SN $2015 \mathrm{cp}$ Revealed by an HST Ultraviolet Imaging Survey. Astrophys. J. 871, 62 (2019).

75. Mattila, S. et al. Early and late time VLT spectroscopy of SN 2001elprogenitor constraints for a type Ia supernova. Astron. Astrophys. 443, 649-662 (2005).

76. Leonard, D. C. Constraining the Type Ia Supernova Progenitor: The Search for Hydrogen in Nebular Spectra. Astrophys. J. 670, 1275-1282 (2007)

77. Lundqvist, P. et al. Hydrogen and helium in the spectra of Type Ia supernovae. Mon. Not. R. Astron. Soc. 435, 329-345 (2013).
78. Shappee, B. J., Stanek, K. Z., Pogge, R. W. \& Garnavich, P. M. No Stripped Hydrogen in the Nebular Spectra of Nearby Type Ia Supernova 2011 fe. Astrophys. J. 762, L5 (2013).

79. Lundqvist, P. et al. No trace of a single-degenerate companion in late spectra of supernovae $2011 \mathrm{fe}$ and 2014J. Astron. Astrophys. 577, A39 (2015).

80. Maguire, K. Type ia supernovae. In Alsabti, A. W. \& Murdin, P. (eds.) Handbook of Supernovae, 1-24 (Springer International Publishing, Cham, 2016).

81. Shappee, B. J. et al. Strong Evidence against a Non-degenerate Companion in SN 2012cg. Astrophys. J. 855, 6 (2018).

82. Sand, D. J. et al. Nebular Spectroscopy of the "Blue Bump" Type Ia Supernova 2017cbv. Astrophys. J. 863, 24 (2018).

83. Tucker, M. A., Shappee, B. J. \& Wisniewski, J. P. No Stripped Companion Material in the Nebular Spectrum of the "Two-Component" Type Ia Supernova ASASSN-18bt. Astrophys. J. 872, L22 (2019).

84. Sand, D. J. et al. Nebular $\mathrm{H} \alpha$ Limits for Fast Declining SNe Ia. Astrophys. J. 877, L4 (2019)

85. Kollmeier, J. A. et al. $\mathrm{H} \alpha$ emission in the nebular spectrum of the Type Ia supernova ASASSN-18tb. Mon. Not. R. Astron. Soc. 486, 30413046 (2019).

86. Vallely, P. J. et al. ASASSN-18tb: a most unusual Type Ia supernova observed by TESS and SALT. Mon. Not. R. Astron. Soc. 487, 23722384 (2019).

87. Parrent, J. T. et al. A Study of Carbon Features in Type Ia Supernova Spectra. Astrophys. J. 732, 30 (2011).

88. Thomas, R. C. et al. Type Ia Supernova Carbon Footprints. Astrophys. J. 743, 27 (2011).

89. Blondin, S. et al. The Spectroscopic Diversity of Type Ia Supernovae. Astron. J. 143, 126 (2012).

90. Folatelli, G. et al. Unburned Material in the Ejecta of Type Ia Supernovae. Astrophys. J. 745, 74 (2012)

91. Silverman, J. M. \& Filippenko, A. V. Berkeley Supernova Ia Program - IV. Carbon detection in early-time optical spectra of Type Ia supernovae. Mon. Not. R. Astron. Soc. 425, 1917-1933 (2012).

92. Maguire, K. et al. Exploring the spectral diversity of low-redshift Type Ia supernovae using the Palomar Transient Factory. Mon. Not. R. Astron. Soc. 444, 3258-3274 (2014)

93. Heringer, E., van Kerkwijk, M. H., Sim, S. A., Kerzendorf, W. E. \& Graham, M. L. Spectral Sequences of Type Ia Supernovae. II. Carbon as a Diagnostic Tool for Explosion Mechanisms. Astrophys. J. 871, 250 (2019).

94. Zhao, X. et al. The Oxygen Features in Type Ia Supernovae and Implications for the Nature of Thermonuclear Explosions. Astrophys. $J$. 826, 211 (2016)

95. Ashall, C. et al. Photometric and spectroscopic observations, and abundance tomography modelling of the Type Ia supernova SN 2014J located in M82. Mon. Not. R. Astron. Soc. 445, $4427-4437$ (2014).

96. Ma, H., Woosley, S. E., Malone, C. M., Almgren, A. \& Bell, J. Carbon Deflagration in Type Ia Supernova. I. Centrally Ignited Models. Astrophys. J. 771, 58 (2013).

97. Brown, P. J., Baron, E., Milne, P., Roming, P. W. A. \& Wang, L. Theoretical Clues to the Ultraviolet Diversity of Type Ia Supernovae. Astrophys. J. 809, 37 (2015).

98. Foley, R. J. et al. Ultraviolet diversity of Type Ia Supernovae. Mon. Not. R. Astron. Soc. 461, 1308-1316 (2016).

99. Maguire, K. et al. Using late-time optical and near-infrared spectra to constrain Type Ia supernova explosion properties. Mon. Not. R. Astron. Soc. 477, 3567-3582 (2018)

100. Seitenzahl, I. R. \& Townsley, D. M. Nucleosynthesis in thermonuclear supernovae. In Alsabti, A. W. \& Murdin, P. (eds.) Handbook of Supernovae, 1-24 (Springer International Publishing, Cham, 2017). Preprint at http://arxiv.org/abs/1704.00415.

101. Maeda, K. et al. An asymmetric explosion as the origin of spectral evolution diversity in type Ia supernovae. Nature 466, 82-85 (2010). 
102. Leonard, D. C., Li, W., Filippenko, A. V., Foley, R. J. \& Chornock, R. Evidence for Spectropolarimetric Diversity in Type Ia Supernovae. Astrophys. J. 632, 450-475 (2005).

103. Wang, L. et al. Premaximum Spectropolarimetry of the Type Ia SN 2004dt. Astrophys. J. 653, 490-502 (2006).

104. Patat, F. et al. VLT spectropolarimetry of the fast expanding type Ia SN 2006X. Astron. Astrophys. 508, 229-246 (2009).

105. Dong, S., Katz, B., Kushnir, D. \& Prieto, J. L. Type Ia supernovae with bimodal explosions are common - possible smoking gun for direct collisions of white dwarfs. Mon. Not. R. Astron. Soc. 454, L61-L65 (2015).

106. Vallely, P. J. et al. Signatures of Bimodality in Nebular Phase Type Ia Supernova Spectra: Indications of White Dwarf Collision Progenitors. Preprint at http://arxiv.org/abs/1902.00037 (2019).

107. van den Bergh, S. The frequency of SN Ia in galaxies of different Hubble type. Publ. Astron. Soc. Pac. 102, 1318-1320 (1990).

108. Mannucci, F. et al. The supernova rate per unit mass. Astron. Astrophys. 433, 807-814 (2005)

109. Sullivan, M. et al. Rates and Properties of Type Ia Supernovae as a Function of Mass and Star Formation in Their Host Galaxies. Astrophys. J. 648, 868-883 (2006).

110. Brown, J. S. et al. The relative specific Type Ia supernovae rate from three years of ASAS-SN. Mon. Not. R. Astron. Soc. 484, 3785-3796 (2019).

111. Smith, M. et al. The SDSS-II Supernova Survey: Parameterizing the Type Ia Supernova Rate as a Function of Host Galaxy Properties. Astrophys. J. 755, 61 (2012).

112. Kistler, M. D., Stanek, K. Z., Kochanek, C. S., Prieto, J. L. \& Thompson, T. A. The Impact of Metallicity on the Rate of Type Ia Supernovae. Astrophys. J. 770, 88 (2013).

113. Graur, O., Bianco, F. B. \& Modjaz, M. A unified explanation for the supernova rate-galaxy mass dependence based on supernovae detected in Sloan galaxy spectra. Mon. Not. R. Astron. Soc. 450, 905-925 (2015).

114. Maoz, D., Mannucci, F. \& Nelemans, G. Observational Clues to the Progenitors of Type Ia Supernovae. Annu. Rev. Astron. Astrophys. 52 107-170 (2014).

115. Graur, O. et al. Type-Ia Supernova Rates to Redshift 2.4 from CLASH The Cluster Lensing And Supernova Survey with Hubble. Astrophys. J. 783, 28 (2014)

116. Frohmaier, C. et al. The volumetric rate of normal type Ia supernovae in the local universe discovered by the Palomar Transient Factory. Mon. Not. R. Astron. Soc. (2019).

117. Branch, D., Romanishin, W. \& Baron, E. Statistical Connections between the Properties of Type IA Supernovae and the B-V Colors of Their Parent Galaxies, and the Value of H0. Astrophys. J. 465, 73 (1996).

118. Hamuy, M. et al. A Search for Environmental Effects on Type Ia Supernovae. Astron. J. 120, 1479-1486 (2000).

119. Johansson, J. et al. SN Ia host galaxy properties from Sloan Digital Sky Survey-II spectroscopy. Mon. Not. R. Astron. Soc. 435, 16801700 (2013)

120. Howell, D. A., Sullivan, M., Conley, A. \& Carlberg, R. Predicted and Observed Evolution in the Mean Properties of Type Ia Supernovae with Redshift. Astrophys. J. 667, L37-L40 (2007).

121. Shen, K. J., Toonen, S. \& Graur, O. The Evolution of the Type Ia Supernova Luminosity Function. Astrophys. J. 851, L50 (2017).

122. Kelly, P. L., Hicken, M., Burke, D. L., Mandel, K. S. \& Kirshner, R. P. Hubble Residuals of Nearby Type Ia Supernovae are Correlated with Host Galaxy Masses. Astrophys. J. 715, 743-756 (2010).

123. Sullivan, M. et al. The dependence of Type Ia Supernovae luminosities on their host galaxies. Mon. Not. R. Astron. Soc. 406, 782-802 (2010).

124. Lampeitl, H. et al. The Effect of Host Galaxies on Type Ia Supernovae in the SDSS-II Supernova Survey. Astrophys. J. 722, 566-576 (2010).

125. Childress, M. et al. Host Galaxy Properties and Hubble Residuals of Type Ia Supernovae from the Nearby Supernova Factory. Astrophys. $J$. 770, 108 (2013).
126. Scolnic, D. M. et al. The Complete Light-curve Sample of Spectroscopically Confirmed SNe Ia from Pan-STARRS1 and Cosmological Constraints from the Combined Pantheon Sample. Astrophys. J. 859, 101 (2018).

127. Brout, D. et al. First Cosmology Results Using SNe Ia from the Dark Energy Survey: Analysis, Systematic Uncertainties, and Validation. Astrophys. J. 874, 150 (2019).

128. Rigault, M. et al. Strong Dependence of Type Ia Supernova Standardization on the Local Specific Star Formation Rate. Preprint at http://arxiv.org/abs/1806.03849 (2018).

129. Jones, D. O. et al. Should Type Ia Supernova Distances Be Corrected for Their Local Environments? Astrophys. J. 867, 108 (2018).

130. Rigault, M. et al. Evidence of environmental dependencies of Type Ia supernovae from the Nearby Supernova Factory indicated by local H $\alpha$. Astron. Astrophys. 560, A66 (2013).

131. Rose, B. M., Garnavich, P. M. \& Berg, M. A. Think Global, Act Local: The Influence of Environment Age and Host Mass on Type Ia Supernova Light Curves. Astrophys. J. 874, 32 (2019).

132. Roman, M. et al. Dependence of Type Ia supernova luminosities on their local environment. Astron. Astrophys. 615, A68 (2018).

133. Kelly, P. L. et al. Distances with $<4 \%$ precision from type Ia supernovae in young star-forming environments. Science 347, 1459-1462 (2015).

134. Childress, M. J., Wolf, C. \& Zahid, H. J. Ages of Type Ia supernovae over cosmic time. Mon. Not. R. Astron. Soc. 445, 1898-1911 (2014).

135. Taubenberger, S. The extremes of thermonuclear supernovae. In Alsabti, A. W. \& Murdin, P. (eds.) Handbook of Supernovae, 157 (Springer International Publishing, Cham, 2017). Preprint at http://arxiv.org/abs/1703.00528

136. Silverman, J. M. et al. Berkeley Supernova Ia Program - I. Observations, data reduction and spectroscopic sample of 582 low-redshift Type Ia supernovae. Mon. Not. R. Astron. Soc. 425, 1789-1818 (2012).

137. Panther, F. H. et al. SN1991bg-like supernovae are associated with old stellar populations. arXiv e-prints arXiv:1904.10139 (2019).

138. Srivastav, S., Anupama, G. C., Sahu, D. K. \& Ravikumar, C. D. SN 2015bp: adding to the growing population of transitional Type Ia supernovae. Mon. Not. R. Astron. Soc. 466, 2436-2449 (2017).

139. Garnavich, P. M. et al. The Luminosity of SN 1999by in NGC 2841 and the Nature of "Peculiar" Type Ia Supernovae. Astrophys. J. 613, $1120-1132$ (2004).

140. Hsiao, E. Y. et al. Carnegie Supernova Project-II: The Near-infrared Spectroscopy Program. Publ. Astron. Soc. Pac. 131, 014002 (2019).

141. Foley, R. J. et al. Type Iax Supernovae: A New Class of Stellar Explosion. Astrophys. J. 767, 57 (2013).

142. Jha, S. W. Type Iax Supernovae. In Alsabti, A. W. \& Murdin, P. (eds.) Handbook of Supernovae, 375 (Springer International Publishing, Cham, 2017). Preprint at http://arxiv.org/abs/1707.01110.

143. Li, W. et al. SN 2002cx: The Most Peculiar Known Type Ia Supernova. Publ. Astron. Soc. Pac. 115, 453-473 (2003).

144. Jha, S. et al. Late-Time Spectroscopy of SN 2002cx: The Prototype of a New Subclass of Type Ia Supernovae. Astron. J. 132, 189-196 (2006).

145. Stritzinger, M. D. et al. Comprehensive observations of the bright and energetic Type Iax SN 2012Z: Interpretation as a Chandrasekhar mass white dwarf explosion. Astron. Astrophys. 573, A2 (2015).

146. Tomasella, L. et al. Optical and near-infrared observations of SN 2014ck: an outlier among the Type Iax supernovae. Mon. Not. R. Astron. Soc. 459, 1018-1038 (2016)

147. McCully, C. et al. Hubble Space Telescope and Ground-based Observations of the Type Iax Supernovae SN 2005hk and SN 2008A. Astrophys. J. 786, 134 (2014).

148. McCully, C. et al. A luminous, blue progenitor system for the type Iax supernova 2012Z. Nature 512, 54-56 (2014).

149. Kromer, M. et al. 3D deflagration simulations leaving bound remnants: a model for 2002cx-like Type Ia supernovae. Mon. Not. R. Astron. Soc. 429, 2287-2297 (2013). 
150. Vennes, S. et al. An unusual white dwarf star may be a surviving remnant of a subluminous Type Ia supernova. Science 357, 680-683 (2017).

151. Raddi, R. et al. Partly burnt runaway stellar remnants from peculiar thermonuclear supernovae. Preprint at http://arxiv.org/abs/1902.05061 (2019)

152. White, C. J. et al. Slow-speed Supernovae from the Palomar Transient Factory: Two Channels. Astrophys. J. 799, 52 (2015).

153. Cao, Y. et al. A strong ultraviolet pulse from a newborn type Ia supernova. Nature 521, 328-331 (2015).

154. Taubenberger, S. et al. [O I] $\lambda \lambda 6300,6364$ in the Nebular Spectrum of a Subluminous Type Ia Supernova. Astrophys. J. 775, L43 (2013).

155. Kromer, M. et al. The peculiar Type Ia supernova iPTF14atg: Chandrasekhar-mass explosion or violent merger? Mon. Not. R. As tron. Soc. 459, 4428-4439 (2016).

156. Howell, D. A. et al. The type Ia supernova SNLS-03D3bb from a super-Chandrasekhar-mass white dwarf star. Nature 443, 308-311 (2006).

157. Taubenberger, S. et al. High luminosity, slow ejecta and persistent carbon lines: SN 2009dc challenges thermonuclear explosion scenarios. Mon. Not. R. Astron. Soc. 412, 2735-2762 (2011).

158. Taubenberger, S. et al. 'Super-Chandrasekhar' Type Ia Supernovae at nebular epochs. Mon. Not. R. Astron. Soc. 432, 3117-3130 (2013).

159. Scalzo, R. A. et al. Probing type Ia supernova properties using bolometric light curves from the Carnegie Supernova Project and the CfA Supernova Group. Mon. Not. R. Astron. Soc. 483, 628-647 (2019).

160. Chen, P. et al. ASASSN-15pz: Revealing Significant Photometric Diversity Among 2009dc-like, Peculiar Type Ia Supernovae. Preprint at http://arxiv.org/abs/1904.03198 (2019).

161. Khan, R., Stanek, K. Z., Stoll, R. \& Prieto, J. L. Super-Chandrasekhar SNe Ia Strongly Prefer Metal-poor Environments. Astrophys. J. 737, L24 (2011).

162. Perets, H. B. et al. The Old Environment of the Faint Calcium-rich Supernova SN 2005cz. Astrophys. J. 728, L36 (2011).

163. Kasliwal, M. M. et al. Calcium-rich Gap Transients in the Remote Outskirts of Galaxies. Astrophys. J. 755, 161 (2012).

164. Lyman, J. D. et al. Environment-derived constraints on the progenitor of low-luminosity Type I supernovae. Mon. Not. R. Astron. Soc. 434, 527-541 (2013).

165. Lunnan, R. et al. Two New Calcium-rich Gap Transients in Group and Cluster Environments. Astrophys. J. 836, 60 (2017).

166. Perets, H. B. et al. A faint type of supernova from a white dwarf with a helium-rich companion. Nature 465, 322-325 (2010).

167. Foley, R. J. Kinematics and host-galaxy properties suggest a nuclear origin for calcium-rich supernova progenitors. Mon. Not. R. As tron. Soc. 452, 2463-2478 (2015).

168. Milisavljevic, D. et al. iPTF15eqv: Multiwavelength Exposé of a Peculiar Calcium-rich Transient. Astrophys. J. 846, 50 (2017).

169. De, K. et al. iPTF 16hgs: A Double-peaked Ca-rich Gap Transient in a Metal-poor, Star-forming Dwarf Galaxy. Astrophys. J. 866, 72 (2018).

170. Li, W. et al. The Unique Type Ia Supernova 2000cx in NGC 524 Publ. Astron. Soc. Pac. 113, 1178-1204 (2001).

171. Candia, P. et al. Optical and Infrared Photometry of the Unusual Type Ia Supernova 2000cx. Publ. Astron. Soc. Pac. 115, 277-294 (2003).

172. Silverman, J. M. et al. SN 2000cx and SN 2013bh: extremely rare, nearly twin Type Ia supernovae. Mon. Not. R. Astron. Soc. 436, 12251237 (2013).

173. Drout, M. R. et al. The Fast and Furious Decay of the Peculiar Type Ic Supernova 2005ek. Astrophys. J. 774, 58 (2013)

174. Sullivan, M. et al. The Subluminous and Peculiar Type Ia Supernova PTF 09dav. Astrophys. J. 732, 118 (2011)

175. Kasliwal, M. M. et al. Rapidly Decaying Supernova 2010X: A Candidate “.Ia” Explosion. Astrophys. J. 723, L98-L102 (2010).

176. Fesen, R. A., Weil, K. E., Hamilton, A. J. S. \& Höflich, P. A. Optical and UV Spectra of the Remnant of SN 1885 (S And) in M31. Astrophys. J. 848, 130 (2017).
177. Maguire, K. et al. PTF10ops - a subluminous, normal-width light curve Type Ia supernova in the middle of nowhere. Mon. Not. R. Astron. Soc. 418, 747-758 (2011).

178. Perets, H. B., Badenes, C., Arcavi, I., Simon, J. D. \& Gal-yam, A. An Emerging Class of Bright, Fast-evolving Supernovae with Low-mass Ejecta. Astrophys. J. 730, 89 (2011).

179. Poznanski, D. et al. An Unusually Fast-Evolving Supernova. Science 327, 58 (2010).

180. Burke, J. et al. FLOYDS Classification of AT 2019ein / ATLAS19ieo as a Young and Peculiar SN Ia. The Astronomer's Telegram 12719 (2019).

181. Jiang, J.-A. et al. A hybrid type Ia supernova with an early flash triggered by helium-shell detonation. Nature 550, 80-83 (2017).

182. De, K. et al. ZTF 18aaqeasu (SN2018byg): A Massive Helium-shell Double Detonation on a Sub-Chandrasekhar-mass White Dwarf. Astrophys. J. 873, L18 (2019)

183. Miller, A. A. et al. Color Me Intrigued: The Discovery of iPTF $16 \mathrm{fnm}$, an SN 2002cx-like Object. Astrophys. J. 848, 59 (2017).

184. Frohmaier, C., Sullivan, M., Maguire, K. \& Nugent, P. The Volumetric Rate of Calcium-rich Transients in the Local Universe. Astrophys. $J$. 858, 50 (2018).

185. Holoien, T. W.-S. et al. The ASAS-SN bright supernova catalogue - I 2013-2014. Mon. Not. R. Astron. Soc. 464, 2672-2686 (2017).

186. Tonry, J. L. et al. ATLAS: A High-cadence All-sky Survey System. Publ. Astron. Soc. Pac. 130, 064505 (2018).

187. Drake, A. J. et al. First Results from the Catalina Real-Time Transient Survey. Astrophys. J. 696, 870-884 (2009).

188. Sand, D., Valenti, S., Tartaglia, L., Yang, S. \& Wyatt, S. An Overview of the D $<40 \mathrm{Mpc}$ One Day Cadence Supernova Search, DLT40. In American Astronomical Society Meeting Abstracts \#231, vol. 231 of American Astronomical Society Meeting Abstracts, 245.11 (2018).

189. Hodgkin, S. T., Wyrzykowski, L., Blagorodnova, N. \& Koposov, S Transient astronomy with the Gaia satellite. Philosophical Trans actions of the Royal Society of London Series A 371, 2012023920120239 (2013).

190. Filippenko, A. V., Li, W. D., Treffers, R. R. \& Modjaz, M. The Lick Observatory Supernova Search with the Katzman Automatic Imaging Telescope. In Paczynski, B., Chen, W.-P. \& Lemme, C. (eds.) IAU Colloq. 183: Small Telescope Astronomy on Global Scales, vol. 246 of Astronomical Society of the Pacific Conference Series, 121 (2001).

191. Walker, E. S. et al. First Results from the La Silla-QUEST Supernova Survey and the Carnegie Supernova Project. Astrophys. J. Suppl. Ser. 219, 13 (2015)

192. Lipunov, V. et al. Master Robotic Net. Advances in Astronomy 2010, $349171(2010)$

193. Wyrzykowski, Ł. et al. OGLE-IV Real-Time Transient Search Acta Astronomica 64, 197-232 (2014).

194. Huber, M. et al. The Pan-STARRS Survey for Transients (PSST) first announcement and public release. The Astronomer's Telegram 7153 (2015)

195. Law, N. M. et al. The Palomar Transient Factory: System Overview, Performance, and First Results. Publ. Astron. Soc. Pac. 121, 1395 (2009).

196. Yang, Z. et al. PTSS: Discovery of a Probable Supernova in SDSS J150350.43+482250.7. The Astronomer's Telegram 8757, 1 (2016).

197. Bellm, E. C. et al. The Zwicky Transient Facility: System Overview, Performance, and First Results. Publ. Astron. Soc. Pac. 131, 018002 (2019).

198. Ivezić, Ž. et al. LSST: From Science Drivers to Reference Design and Anticipated Data Products. Astrophys. J. 873, 111 (2019). 
Correspondence Correspondence and requests for materials should be addressed to S.W.J. (saurabh@physics.rutgers.edu).

Acknowledgements We thank Chris Burns, Régis Cartier, Melissa Graham, Eric Hsiao, David Jones, Wenxiong Li, Mikael Rigault, and Stefan Taubenberger for providing figures used here. We are grateful to Ryan Foley, Avishay Gal-Yam, Peter Nugent, Ken Shen, and the anonymous referee for comments and helpful suggestions. We also thank Fiona Panther for alerting us to the Ph.D. thesis of Titus Pankey, Jr. as the genesis of a radioactive nickel-56 power source for the luminosity of supernovae. Support for this review was provided in part by US NSF award AST-1615455 (S.W.J.), H2020/ERC grant 758638 (K.M), and FP7 EU/ERC grant 615929 (M.S.).

Author Contributions S.W.J. wrote the introduction and thermonuclear zoo section of the article. K.M. wrote the section on SN Ia observations, and M.S. wrote the section on SN Ia environments. All authors discussed and edited the text.

Competing Interests The authors declare that they have no competing financial interests. 